\title{
Thermopower and Surface Magnetic Characterization of Ni Thin Layers
}

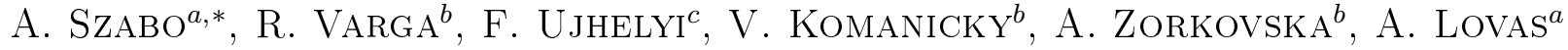

${ }^{a}$ Dept. of Automob. and Vehicle Manuf., Budapest Univ. of Techn, Stoczek u. 2. 1111 Budapest, Hungary

${ }^{b}$ Dept. of Cond. Mat. Phys., Institute of Phys. P.J. Šafárik University, Park Angelinum 904154 Košice, Slovakia

${ }^{c}$ Dept. of Atomic Phys., Budapest Univ. of Techn., Budafoki u. 8. 1111 Budapest, Hungary

\begin{abstract}
Thermoelectric power and surface magnetic characterization of $\mathrm{Ni}$ evaporated layers will be presented, with the comparison with the bulk Ni material. The bulk electrical and magnetic properties are developed gradually, approaching the bulk properties, as the layer thickness increases. Below the thickness of $180 \mathrm{~nm}$, the evaporated layers are amorphous. The epitaxial stresses can appear in both, the anomaly observed in the thermopower and the surface coercivity measurements.
\end{abstract}

DOI: 10.12693 /APhysPolA.126.204

PACS: 72.15.Jf; 73.50.Lw; 75.70.-i; 68.55.jm

\section{Introduction}

While the Kerr effect [1] is originally used for the study of surface magnetic properties, the Seebeck phenomenon, (which has a central role in the thermopower measurements) was originally defined for bulk, macroscopic conductors [2].

Recently, the thermopower measurement is applied in non-destructive material testing and also in thin layer characterization [3]. In spite of the reported applications several details are not clear, especially in the field of the possible crossover effects, arising from the mechanically deformed surface layers and the penetration depth of the thermally activated environment, which is inherently developed during the thermopower measurements. In order to control the actual layer thickness, which actually participates in the measurement process, and also to follow the appearance of "bulk properties", layers with varying thicknesses were produced by the PVD process. Ni samples were prepared, because the characteristics of its magnetic properties are well known.

In this paper an experimental effort is made, in order to recognize and separate the thin layer effects and the bulk effects, which take part in the thermopower measurements.

\section{Experiment}

Materials used are the high purity $(4 \mathrm{~N})$ evaporated $\mathrm{Ni}$ layers (on glassy substrates) and bulk Ni sheets both in cold rolled and normalized state. Details of $S(\Delta T)$ are described in Refs.[4]

\section{Results}

The application of thermopower measurements to the characterization of bulk system is based on the obvious supposition, that the contribution of thin, surface layer is often negligible. For the success of more quantitative

*corresponding author; e-mail: szabo@kgtt.bme.hu appreciation of surface effects, the comparison with an independent, but also surface sensitive method seems to be fruitful. Such is the surface magnetization measurement, which is also performed on the same ferromagnetic material $(\mathrm{Ni})$.

\subsection{Thin layer effects in the thermopower and surface magnetic characterization}

Most of the physical and chemical properties characteristic to the bulk phases exhibit strong size dependence in the nanometer-scale dimension. This is the central phenomenon of the "size related type of metastability" which is also reflected in the thickness dependence of Seebeck coefficient, $S(\Delta T)$, when it is compared to the bulk sample. This is clearly depictured in the Fig. 1.

The curves gradually shift to negative direction with slightly increasing slope, when the layer thickness increases. The curve for bulk $\mathrm{Ni}$ is also plotted.

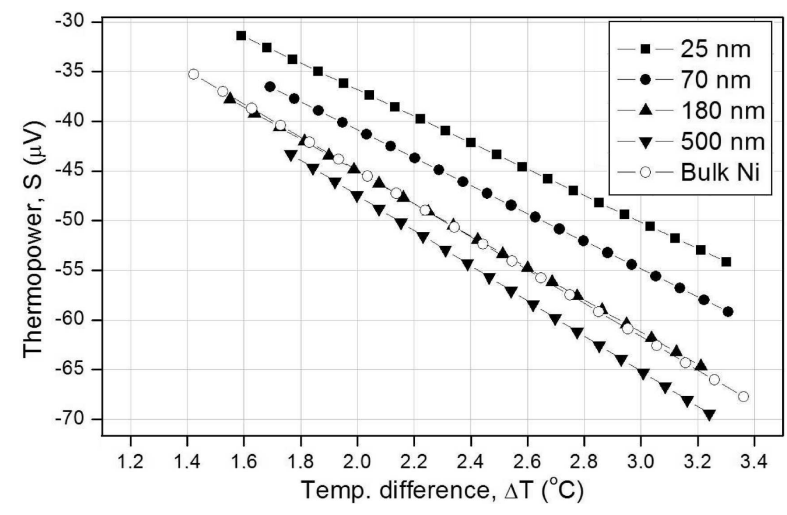

Fig. 1. The $S(\Delta T)$ plots for Ni and Ni thin layers with varying thickness.

The sign and slope are in agreement with the earlier observation [3]. Consequently, typical size and structural effects are reflected in this shift and also in the varying slope of the curves. Similar thickness dependence was 
also detected in (non-ferromagnetic Ti-layers: the evaporated layers represent a nanometer scale extension along the thickness, consequently the bulk transport properties (including the thermopower) are gradually altered due to the modification of Fermi level.

\subsection{Structural and magnetic characterization of Ni-layers}

As the X-ray pattern shows, (see Fig. 2) the $25 \mathrm{~nm}$ and $70 \mathrm{~nm}$ thick layers are fully amorphous, but some crystalline traces can already be detected in the $180 \mathrm{~nm}$ thick layer. The $500 \mathrm{~nm}$ thick layer is fully crystalline.

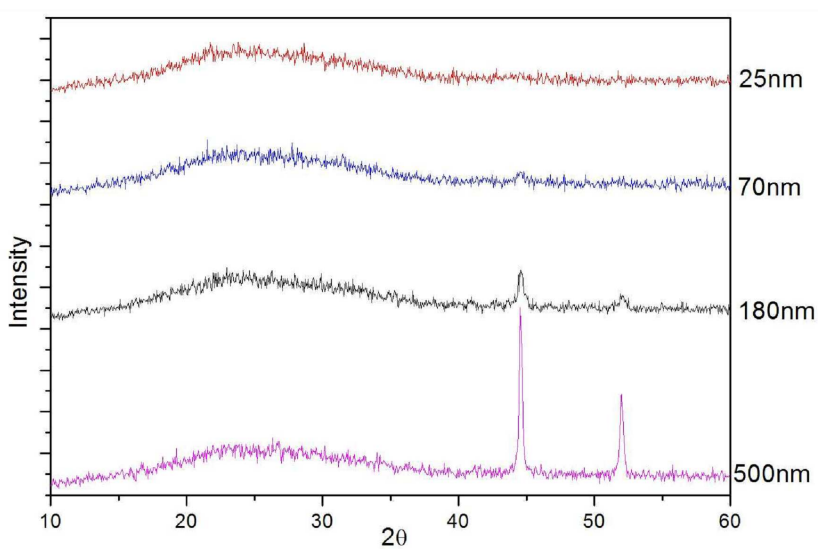

Fig. 2. X-diffractogram on Ni-thin layers.

\subsection{The thickness dependence of magnetization process and coercivity}

The coercivity of amorphous (thinner) $\mathrm{Ni}$ films is slightly higher than that, for the $500 \mathrm{~nm}$, fully crystalline sample, however the difference is not significant (see Table). The higher coercivity of thin layers (either the amorphous or the crystalline ones) compared to that for the bulk sample, points to the significance of epitaxial stress evolution during the layer formation. The glassy substrate structure exerts an epitaxial force on the layers, which is sustained during the whole growth process.

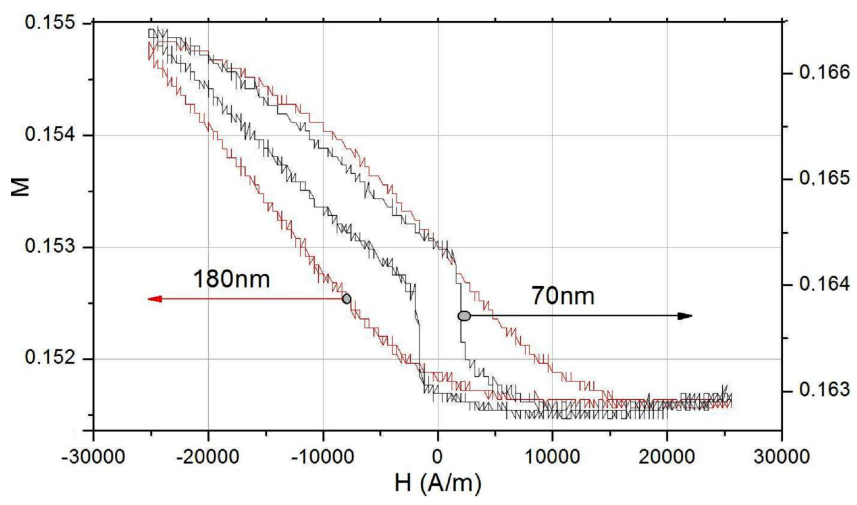

Fig. 3. Surface Hysteresis loops of $\mathrm{Ni}$ thin layers (70 $\mathrm{nm}$ and $180 \mathrm{~nm}$ layer thickness).
TABLE

Coercivity versus the layer thickness in evaporated $\mathrm{Ni}$ thin films and, in bulk $\mathrm{Ni}$ sample.

\begin{tabular}{c|c}
\hline \hline Layer thickness $(\mathrm{nm})$ & Coercivity $(\mathrm{A} / \mathrm{m})$ \\
\hline 25 & 5854 \\
70 & 6159 \\
180 & 5632 \\
500 & 5150 \\
bulk & 1850
\end{tabular}

The shape of magnetization $(M)$ curves changes with the growing thickness of the layer. While $M$ exhibits discontinuity for the thinner samples, it turns to be continuous with increasing layer thickness (see Fig. 3).

Because the penetration depth of the thermal gradient during the thermopower measurements is commensurable, or even higher than the investigated layer thickness, the transformed (or deformed) surface layers may have a serious influence on the measured value of $S$. The conclusion is the same for surface magnetic characterization of the same series of evaporated Ni-samples.

\section{Conclusions}

1. The electronic structure of bulk Ni suffers dramatic change in the scale of $500 \mathrm{~nm}$ layer thickness, which is reflected in the thermopower characteristics of the samples.

2. Below the thickness of $80 \mathrm{~nm}$, the layers are amorphous. The amorphous structure is the consequence of epitaxial stress evolution, dictated by the glassy substrate.

3. The existence of epitaxial stress evolution in the evaporated films is also supported by the high level of surface coercivity, obtained from the Kerr-effect measurements.

\section{Acknowledgments}

This work was supported by the project OTKA K109271

\section{References}

[1] A. Hubert, R. Schafer, Magnetic Domains, Springer Verlag, Berlin-Heidelberg-New York, p. 24, 1998 (ISBN 3-540-64108-4).

[2] N.F. Mott, Electronic processes in non-crystalline materials, Oxford University Press, 2012 ISBN: 0199645337.

[3] A.D. Avery, R. Sultan, D. Bassett, D. Wei, B.L. Zink, Phys. Rev. B 83, 100401(R) (2011).

[4] A. Szabó, J. Kovac, A. Lovas, Perner's Contacts VI, 181 (2011). 